\title{
Comparison of Anti-Obesity Effect between Two Types of Syrup Containing Rare Sugars in Wistar Rats
}

\author{
Masaru OCHIAI ${ }^{1}$, Kohei MisAKI ${ }^{1}$, Takako YAMADA ${ }^{2}$, Tetsuo IIDA ${ }^{2}$, \\ Kazuhiro OKUMA ${ }^{2}$ and Tatsuhiro MATSUO ${ }^{1, *}$ \\ ${ }^{1}$ Faculty of Agriculture, Kagawa University, 2393 Ikenobe, Miki, Kita, Kagawa 761-0795, Japan \\ ${ }^{2}$ Research and Development, Matsutani Chemical Industry Co., Ltd., 5-3 Kita-Itami, \\ Itami, Hyogo 664-8508, Japan
}

(Received December 11, 2016)

\begin{abstract}
Summary D-Allulose-containing rare sugar sweeteners have been categorized into two types, rare sugar syrup (RSS), consisting of 4 rare monosaccharides, and modified glucose syrup (MGS), rich in D-allulose, which was previously referred to D-psicose. The anti-obesity effect of RSS and D-allulose has been already clarified, but that of rare monosaccharides other than D-allulose in RSS has not yet been well understood. Here, we investigated and compared the anti-obesity effect of RSS and MGS in rats. Male Wistar rats were divided into 4 dietary groups: a high-sucrose control diet group (S), a high-fructose corn syrup diet group (HFCS), an RSS diet group (RSS), and an MGS diet group (MGS). RSS significantly suppressed abdominal adipose tissue weight and total body fat accumulation in comparison to sucrose. On the other hand, MGS reduced body weight gain, but not abdominal fat accumulation, relative to sucrose. The weight of the liver and kidneys was significantly higher in the RSS and MGS groups than in the S and HFCS groups, but serum biochemical parameters and hepatic lipids contents were not significantly different among the groups. The present study shows that two types of D-allulose-containing rare sugar sweeteners can suppress body fat accumulation or weight gain in a different manner and that RSS could be used as more effective sweeteners in place of sucrose and HFCS to maintain healthy body weight.
\end{abstract}

Key Words rare sugar sweetener, modified glucose syrup, D-allulose, fat accumulation, rat

High fructose corn syrup (HFCS), which is made by isomerizing D-glucose to D-fructose, has been used in a wide range of food and beverage. The rise in HFCS consumption has been continuously reported to be associated with the growing risk of obesity and diabetes in the United States $(1,2)$. In 2015, the World Health Organization (WHO) recommended that adults and children should reduce their daily intake of free sugars to less than $5 \%$ of their total energy intake (3). Therefore, much more attention has recently been paid to non- or low-calorie sweeteners to maintain healthy body weight and prevent obesity (4). On the other hand, studies have reported that some conventional non- or low-calorie sweeteners such as aspartame and saccharin increase body weight and food consumption, and induce lower thermogenesis and glucose intolerance as adverse effects (5-7).

In order to avoid the adverse effects described above, we have developed rare sugar sweeteners. Among rare sugar sweeteners, D-allulose (D-ribo-2-hexulose), which was previously referred to D-psicose, has recently received much attention as a potential anti-diabetic and anti-obesity ingredient ( $8-15)$, and has not shown adverse effects that are observed in conventional non- or

\footnotetext{
*To whom correspondence should be addressed.

E-mail:matsuo@ag.kagawa-u.ac.jp
}

low-calorie sweeteners.

There have been two methods for producing D-allulose-containing rare sugar sweetener from D-fructose through an isomerization process, using alkali hydrolysis or a microbially-derived isomerizing enzyme. In the latter method, D-glucose derived from cornstarch is isomerized to D-fructose by xylose isomerase, and then is further epimerized to D-allulose by D-allulose 3-epimerase, which has greater substrate specificity to D-allulose than D-tagatose 3-epimerase (14). The enzymatic method converts D-fructose to D-allulose at a high conversion rate $(\sim 25 \%)$, resulting in pure and costly D-allulose. In contrast, D-allulose produced from HFCS by alkali hydrolysis is present at the level of $\sim 6 \%$ in rare sugar syrup (RSS), which has been sold as a less expensive sweetener in local markets. We have reported that RSS shows no adverse health effects in rats or humans (16) and suppresses body fat accumulation in rats (17). We also showed that RSS reduced abdominal fat accumulation and serum glucose, insulin, and leptin levels in rats compared to HFCS (18). In our previous study, D-allulose suppressed lipogenic enzymatic activities in the liver compared to glucose or fructose in rats (19, 20). Thus, RSS containing D-allulose seem to be effective in preventing body fat accumulation and obesity partly via lowered lipogenic enzymatic activities. However, D-allulose may not be the sole monosaccharide in 


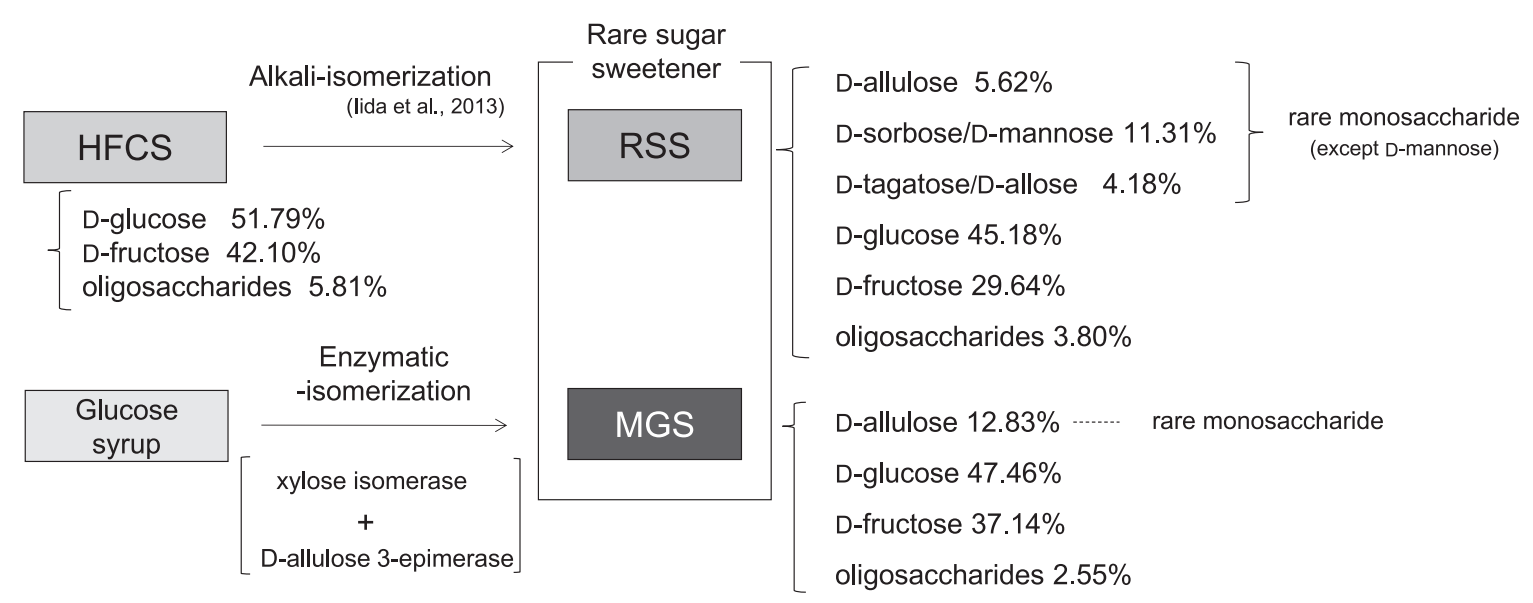

Fig. 1. Production method of RSS and MGS and their sugar compositions. Rare sugar syrup (RSS) and modified glucose syrup (MGS) are categorized as rare sugar sweeteners. RSS is produced by alkali-isomerization from high-fructose corn syrup (HFCS) as described in Iida et al. (18), and contains D-allulose, D-sorbose, D-tagatose, and D-allose as a rare monosaccharide. MGS is produced by the enzymatic reaction (xylose isomerase and D-allulose 3-epimerase) from glucose syrup, and contains mainly D-allulose as a rare saccharide. These two enzymes were successfully reacted in a single step to produce the MGS from glucose syrup more effectively in the present study.

RSS to reduce body fat accumulation because other rare monosaccharides in addition to D-allulose are also present in RSS.

In the present study, we prepared a new rare sugar sweetener rich in D-allulose with the modified enzymatic reaction using glucose syrup as described above, and referred to it as a new modified glucose syrup (MGS). Then, we compared the anti-obesity property of the two types of D-allulose-containing rare sugar sweeteners (RSS and MGS) in rats.

\section{Materials and Methods}

Preparation of MGS and RSS. Both RSS and MGS are categorized as rare sugar sweeteners. MGS was produced from glucose syrup by the patented method developed by Matsutani Chemical Industry Co., Ltd. (Hyogo, Japan) as described in Fig. 1. Briefly, RSS is produced by alkali-isomerization from HFCS as described by lida et al. (18), and contains D-allulose, D-sorbose, D-tagatose, and D-allose as rare monosaccharides. MGS is produced by the enzymatic reaction (xylose isomerase and D-allulose 3-epimerase) from glucose syrup, and contains mainly D-allulose as its rare monosaccharide. Two enzymatic steps for producing D-allulose from glucose were combined to one step by mixing two enzymes. HFCS, RSS, and MGS were supplied by Matsutani Chemical Industry Co., Ltd. The solid concentration of HFCS, RSS, and MGS used in the present study was 69,75 , and $75 \%$ $(\mathrm{g} / 100 \mathrm{~g})$, respectively. MGS mainly consists of D-glucose, D-fructose, D-allulose, and oligosaccharides while RSS mainly consists of D-glucose, D-fructose, D-allulose, D-mannose, D-sorbose, D-tagatose, D-allose, and oligosaccharides. HFCS contains D-glucose, D-fructose, and oligosaccharides.

Reagents. Chemical reagents were purchased from Wako Pure Chemical Industries, Ltd. (Osaka, Japan) or Nacalai tesque, Inc. (Kyoto, Japan). Powdered fat (Magic fat 200) was donated from Miyoshi Oil \& Fat Co., Ltd. (Tokyo, Japan). A mineral mixture and vitamin mixture were purchased from Oriental Yeast Co., Ltd. (Tokyo, Japan). Casein, sucrose, cellulose, and cornstarch were purchased from Fonterra Co. (Auckland, New Zealand), Mitsui Sugar Co., Ltd. (Tokyo, Japan), Nippon Paper Industries Co., Ltd. (Tokyo, Japan), and Oji Cornstarch Co., Ltd. (Tokyo, Japan), respectively.

Animal treatment. Animal experiments were approved by the Experimental Animal Care Committee of Kagawa University (Approval No. 2014-30). Thirtyfour male Wistar rats (3 wk of age) were purchased from Japan SLC, Inc. (Shizuoka, Japan). Rats were individually housed in stainless steel cages and acclimatized at $22 \pm 2^{\circ} \mathrm{C}$ under $12 \mathrm{~h} \mathrm{light/dark} \mathrm{cycle} \mathrm{(lights} \mathrm{on} \mathrm{from}$ 08:00 to 20:00). Rats were given water and an MF diet (Oriental Yeast Co., Ltd.) ad libitum for 7 d. Rats were then divided into four dietary groups: rats fed a highsucrose control diet (S, $n=8)$, an HFCS diet (HFCS, $n=8$ ), an RSS diet (RSS, $n=8$ ), and an MGS diet (MGS, $n=10$ ). The composition of the diets is shown in Table 1. The levels of RSS and MGS were within the range that does not cause diarrhea, based on our previous experiment (Misaki et al., unpublished data). Rats were fed the diets for $8 \mathrm{wk}$, and body weight and dietary food intake were monitored daily. At the end of the feeding period, rats were fasted for $12 \mathrm{~h}$ and subsequently sacrificed by decapitation. Serum, liver, kidney, abdominal adipose tissues, and muscles were stored at $-80^{\circ} \mathrm{C}$ until biochemical analyses. Other organs, tissues, head, legs, and tail were removed, and remaining parts were defined as the carcass. Carcass samples were also stored at $-20^{\circ} \mathrm{C}$ until analyses.

Biochemical analysis. The levels of serum glucose, triacylglycerol (TG), and total cholesterol were measured using commercial kits (Wako Pure Chemical Industries, Ltd.). The serum insulin level was measured using 
Table 1. Composition of experimental diet ( $\mathrm{g} / \mathrm{kg}$ diet).

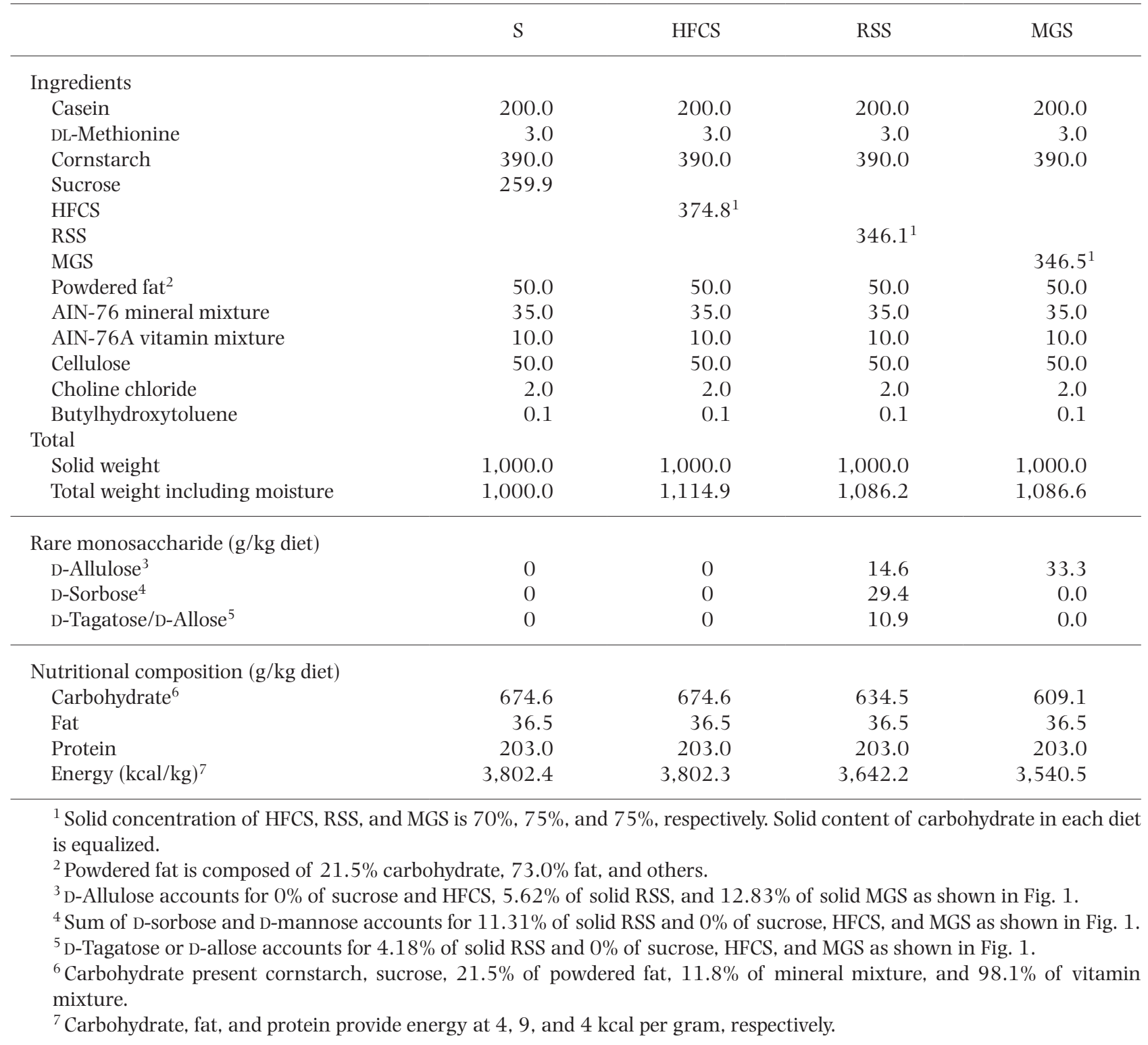

a commercial kit (Shibayagi Co., Ltd., Gunma, Japan). Total lipids in the liver were extracted as described by Folch et al. (21). The levels of hepatic TG and cholesterol were measured as described in serum analyses. The fat contents of the carcass and total body were measured and calculated as described previously (12). Briefly, a carcass sample softened by autoclave treatment $\left(121^{\circ} \mathrm{C}\right.$, $90 \mathrm{~min}$ ) was ground using a food mixer, and debris of bone was fully removed in order to obtain a carcass meat sample. Crude lipids in the carcass meat sample were extracted by petroleum ether $\left(70^{\circ} \mathrm{C}\right)$ with a Soxhlet extractor, and then extracted crude lipids were weighed to quantify the carcass fat weight.

Statistical analysis. Data are expressed as means \pm SE $(n=8-10)$. Statistical analysis of differences among the groups was performed by one-way ANOVA with posthoc Tukey-Kramer tests. A difference at $p<0.05$ was considered to be statistically significant. Statistical analyses were carried out using a commercially available statistical package (Excel Statistics 2015, SSRI, Tokyo,
Japan).

\section{Results and Discussion}

As shown in Table 2, abdominal adipose tissues weight and total body fat percentage were significantly lower in the RSS group, but not the MGS group, than in the $S$ group. These results were consistent with our previous study (18) indicating that dietary RSS suppressed fat accumulation in abdominal adipose tissues of rats. RSS contains D-allulose, D-tagatose, D-sorbose, and D-allose as rare monosaccharides while MGS consists of D-allulose. Thus, the reason why RSS, but not MGS, induced the anti-obese action is probably that each rare sugar other than D-allulose in RSS has a different function, such as fermentability and the effects on glucokinase described below.

RSS contains D-sorbose, D-tagatose, and D-allose as rare monosaccharides in addition to D-allulose. D-Sorbose has been reported to inhibit intestinal $\alpha$-glucosidase activity and postprandial hyperglycemia in rats 
Table 2. Body weight, food intake, tissue weights, serum biochemical parameter, and hepatic lipids content.

\begin{tabular}{|c|c|c|c|c|}
\hline Group & $S$ & HFCS & RSS & MGS \\
\hline D-Allulose content in diet (\%) & 0 & 0 & 1.5 & 3.3 \\
\hline Number of rats & 8 & 8 & 8 & 10 \\
\hline \multicolumn{5}{|l|}{ Body weight and food intake } \\
\hline Body weight (initial) (g) & $67.4 \pm 1.0$ & $67.2 \pm 1.1$ & $67.4 \pm 1.3$ & $67.4 \pm 1.4$ \\
\hline Body weight (final) (g) & $281 \pm 6.2$ & $268 \pm 6.6$ & $267 \pm 5.7$ & $254 \pm 7.3^{*}$ \\
\hline Food intake $(\mathrm{g} / \mathrm{rat} / \mathrm{d})^{1}$ & $16.4 \pm 0.3$ & $16.2 \pm 0.6$ & $16.5 \pm 0.4$ & $16.0 \pm 0.4$ \\
\hline Food efficiency (g BW/g) & $0.23 \pm 0.00$ & $0.22 \pm 0.00$ & $0.21 \pm 0.00^{*}$ & $0.20 \pm 0.00^{* * *}$ \\
\hline \multicolumn{5}{|c|}{ Weight of tissues and organs, and fat weight } \\
\hline Liver (mg/g BW) & $32.3 \pm 0.4$ & $31.1 \pm 0.5$ & $34.8 \pm 0.7^{* \# \# \#}$ & $34.9 \pm 0.4^{* * \# \# \#}$ \\
\hline Kidney (mg/g BW) & $6.1 \pm 0.1$ & $6.3 \pm 0.1$ & $7.1 \pm 0.1^{* * * \# \#}$ & $7.9 \pm 0.1^{* * * \# \# \# \$ \$ \$}$ \\
\hline \multicolumn{5}{|c|}{ Abdominal adipose tissues (mg/g BW) } \\
\hline Perirenal & $28.3 \pm 1.5$ & $26.8 \pm 1.7$ & $21.8 \pm 1.3^{*}$ & $24.9 \pm 1.5$ \\
\hline Epididymal & $34.1 \pm 1.0$ & $31.6 \pm 1.7$ & $25.7 \pm 1.5^{* *}$ & $30.4 \pm 1.5$ \\
\hline Mesenteric & $24.6 \pm 1.0$ & $21.8 \pm 1.3$ & $18.5 \pm 1.4^{*}$ & $20.9 \pm 1.2$ \\
\hline Total & $86.9 \pm 3.3$ & $80.2 \pm 4.5$ & $66.0 \pm 4.1^{* *}$ & $76.2 \pm 4.1$ \\
\hline \multicolumn{5}{|l|}{ Muscles (mg/g BW) } \\
\hline Soleus & $0.72 \pm 0.02$ & $0.73 \pm 0.02$ & $0.72 \pm 0.01$ & $0.71 \pm 0.02$ \\
\hline Plantaris & $1.86 \pm 0.03$ & $1.94 \pm 0.03$ & $1.95 \pm 0.04$ & $1.94 \pm 0.03$ \\
\hline Gastrocnemius & $8.88 \pm 0.14$ & $9.16 \pm 0.12$ & $9.34 \pm 0.17$ & $9.36 \pm 0.18$ \\
\hline Total & $11.5 \pm 0.17$ & $11.8 \pm 0.16$ & $12.0 \pm 0.21$ & $12.0 \pm 0.22$ \\
\hline Carcass fat weight (\%) & $16.7 \pm 0.7$ & $16.9 \pm 1.6$ & $13.3 \pm 0.8$ & $15.5 \pm 1.0$ \\
\hline Total body fat weight (\%) & $16.4 \pm 0.6$ & $15.8 \pm 1.1$ & $12.6 \pm 0.7^{*}$ & $14.7 \pm 0.8$ \\
\hline \multicolumn{5}{|l|}{ Serum biochemical component } \\
\hline Triacylglycerol (mg/100 mL) & $197 \pm 19.0$ & $137 \pm 9.3$ & $172 \pm 15.2$ & $183 \pm 16.4$ \\
\hline Total cholesterol (mg/100 mL) & $93.5 \pm 5.4$ & $95.5 \pm 4.6$ & $94.3 \pm 5.1$ & $93.8 \pm 2.9$ \\
\hline Glucose $(\mathrm{mg} / 100 \mathrm{~mL})$ & $137 \pm 5.8$ & $134 \pm 4.6$ & $135 \pm 5.6$ & $139 \pm 4.9$ \\
\hline Insulin $(\mathrm{ng} / \mathrm{mL})$ & $1.9 \pm 0.4$ & $1.7 \pm 0.2$ & $2.2 \pm 0.3$ & $1.9 \pm 0.2$ \\
\hline \multicolumn{5}{|l|}{ Liver lipid contents } \\
\hline Triacylglycerol (mg/g tissue) & $73.2 \pm 8.3$ & $92.4 \pm 8.1$ & $67.5 \pm 7.2$ & $70.6 \pm 6.8$ \\
\hline Cholesterol (mg/g tissue) & $12.7 \pm 0.9$ & $13.1 \pm 0.2$ & $15.1 \pm 0.6$ & $14.1 \pm 0.7$ \\
\hline
\end{tabular}

Data represent mean \pm SE for $8-10$ rats per group.

Statistically significant differences were evaluated by one-way ANOVA and the Tukey-Kramer test. A difference of $p<0.05$ was considered to be statistically significant. ${ }^{* * *, * * *} p<0.05,0.01,0.001$ compared with the $S$ group, respectively. $\# \#$.\#\#\# $p<0.01,0.001$ compared with the HFCS group, respectively. ${ }^{\$ \$} p<0.001$ compared with the RSS group.

S, sucrose; HFCS, high-fructose corn syrup; RSS, rare sugar syrup; MGS, modified glucose syrup. ${ }^{1}$ Food intake is shown as solid weight.

(22). Our previous study (23) has shown that D-sorbose improves serum insulin level and increases cecum weight in rats. However, the anti-obesity effects of D-sorbose have not yet been reported. D-Tagatose prevented diabetes and obesity (24). In the review (24), D-tagatose-1-phosphate, a D-tagatose metabolite, has been reported to induce the translocation of glucokinase and inhibit glycogen phosphorylase, which in turn promotes hepatic glycogen synthesis. Bär (25) also suggested that tagatose-induced hepatic hypertrophy was accompanied with glycogen deposition, but not lipid deposition. In addition, D-tagatose has been shown to exert an inhibitory activity against intestinal $\alpha$-glucosidase, improving postprandial hyperglycemia (24). However, the contribution of dietary D-tagatose (at most around $1 \%$ in the RSS diet) to the anti-obesity effect induced by RSS is not clear. On the other hand, D-allose, another rare monosaccharide in RSS, has not been studied for its anti-obesity effect although it has various physiological functions such as anti-cancer and anti-oxidative functions (26). Thus, how each rare monosaccharide in RSS contributes to the anti-obese effect should be addressed in future studies.

On the other hand, MGS had a smaller effect on abdominal fat compared to RSS (Table 2), although MGS contains twice more D-allulose than RSS in each $\operatorname{diet}(3.3 \%$ vs. $1.5 \%)$. In animal studies investigating the anti-obesity effects of D-allulose, D-allulose content in the diet was mainly set at the level of $3 \%(27)$ or $5 \%$ $(11,12,15,28)$, although the carbohydrate source in the diets and the animal model varied depending on studies. Our previous study (28) reported that a 5\% D-allulose diet for $8 \mathrm{wk}$ significantly suppressed body fat accumulation and increased energy expenditure in rats fed a sucrose-based diet. Nagata et al. (27) reported that a 3\% D-allulose diet for $4 \mathrm{wk}$ favorably altered fat metabolism by increasing energy expenditure and fat oxidation in rats fed a starch-based diet. Considering the 
previous findings, D-allulose content (3.3\%) in the MGS diet is supposed to be sufficient to improve fat metabolism. Although reasons why the anti-obese effects of MGS are not clear at present, as mentioned above, additional effect of rare sugars other than D-allulose in RSS may be anticipated. Thus, several kinds of rare monosaccharides in RSS may function mutually, resulting in suppressed body fat accumulation in the RSS group as also shown in our previous study (18).

Final body weight was significantly lower in the MGS group, but not in the RSS group, than in the S group. Food intake was not different among the groups, while food efficiency was significantly lower in the RSS and MGS groups than in the $\mathrm{S}$ group. Muscle weight was comparable among the groups. Thus, MGS suppressed body weight gain without decreasing food intake or muscle mass. Reasons for lower food efficiency in the MGS group without a change in abdominal adipose tissue weight should be addressed in future studies.

Serum biochemical parameters and hepatic lipid contents were not significantly different among the groups although RSS and MGS lowered the abdominal fat percentage and body weight, respectively. Since RSS and MGS are prepared from HFCS and glucose, respectively, and are high in glucose and fructose, these nonrare sugar monosaccharides might have lessened the lipid-lowering effect of D-allulose and other rare monosaccharides. When compared to the starch-based diet group, RSS supplementation for 8 wk reduced hepatic TG accumulation as well as intra-abdominal fat accumulation in rats (18). MGS, which contains D-allulose as its sole rare sugar, therefore could reduce hepatic lipids accumulation when a starch-based diet is supplied, which requires further studies.

Regarding its safety as a food additive, D-allulose was approved as generally recognized as safe (GRAS Notice No. GRN 498) and is allowed to be used as a form of RSS in a variety of foods and dietary supplements. As shown in Table 2, liver and kidney weight was significantly higher in the RSS and MGS groups than in the S and HFCS groups. In addition, kidney weight was significantly higher in the MGS group than in the RSS group. In our previous sub-chronic toxicity study using rats, administration of D-allulose at the level of 3\% in the diet also showed weight gain of the liver and kidneys, but did not show any adverse health effects (29). Since, similar to D-tagatose, hepatic glycogen accumulation induced by D-allulose has been reported (10), enlarged liver is likely to be due to increased glycogen content. As Tsukamoto et al. (30) revealed that about $37 \%$ of administered D-allulose was excreted via urine at $120 \mathrm{~min}$ after administration, a higher load of D-allulose on kidney function would be also expected in rats fed RSS or MGS, probably resulting in kidney weight gain.

In conclusion, MGS suppressed body weight gain and RSS suppressed abdominal tissue weight and total fat accumulation, indicating that RSS exerts an anti-obesity effect. RSS appears to be a well-balanced rare sugar sweetener to reduce body fat weight more and shows less kidney weight gain compared to MGS. In addition to the fact that RSS is less expensive than MGS, these findings indicate that RSS could be used as a low-calorie sweetener to provide an anti-obesity effect in place of sucrose and HFCS.

\section{REFERENCES}

1) Elliott SS, Keim NL, Stern JS, Teff K, Havel PJ. 2002. Fructose, weight gain, and the insulin resistance syndrome. Am J Clin Nutr 76: 911-922.

2) Gross LS, Li L, Ford ES, Liu S. 2004. Increased consumption of refined carbohydrates and the epidemic of type 2 diabetes in the United States: an ecologic assessment. Am J Clin Nutr 79: 774-779.

3) World Health Organization. 2015. Sugars Intake for Adults and Children. World Health Organization, Geneva, Switzerland.

4) Roberts A. 2016. The safety and regulatory process for low calorie sweeteners in the United States. Physiol Behav 164: 439-444.

5) Kuk JL, Brown RE. 2016. Aspartame intake is associated with greater glucose intolerance in individuals with obesity. Appl Physiol Nutr Metab 41: 795-798.

6) Fowler SP. 2016. Low-calorie sweetener use and energy balance: Results from experimental studies in animals, and large-scale prospective studies in humans. Physiol Behav 164: 517-523.

7) Foletto KC, Melo Batista BA, Neves AM, Feijó MF, Ballard CR, Ribeiro MF, Bertoluci MC. 2016. Sweet taste of saccharin induces weight gain without increasing caloric intake, not related to insulin-resistance in Wistar rats. Appetite 96: 604-610.

8) Hayashi N, Iida T, Yamada T, Okuma K, Takehara I, Yamamoto T, Yamada K, Tokuda M. 2010. Study on the postprandial blood glucose suppression effect of D-psicose in borderline diabetes and the safety of long-term ingestion by normal human subjects. Biosci Biotechnol Biochem 74: 510-519.

9) Baek SH, Park SJ, Lee HG. 2010. D-Psicose, a sweet monosaccharide, ameliorate hyperglycemia, and dyslipidemia in C57BL/6J db/db mice. J Food Sci 75: H49-H53.

10) Hossain A, Kitagaki S, Nakano D, Nishiyama A, Funamoto Y, Matsunaga T, Tsukamoto I, Yamaguchi F, Kamitori K, Dong Y, Hirata Y, Murao K, Toyoda Y, Tokuda M. 2011. Rare sugar D-psicose improves insulin sensitivity and glucose tolerance in type 2 diabetes Otsuka LongEvans Tokushima Fatty (OLETF) rats. Biochem Biophys Res Commun 405: 7-12.

11) Chung YM, Hyun LJ, Youl KD, Hwang SH, Hong YH, Kim SB, Lee JS, Park HC. 2012. Dietary D-psicose reduced visceral fat mass in high-fat diet-induced obese rats. J Food Sci 77: H53-H58.

12) Ochiai $M$, Nakanishi $Y$, Yamada T, Iida T, Matsuo $T$. 2013. Inhibition by dietary D-psicose of body fat accumulation in adult rats fed a high-sucrose diet. Biosci Biotechnol Biochem 77: 1123-1126.

13) Hossain A, Yamaguchi F, Hirose $K$, Matsunaga $T$, Sui L, Hirata Y, Noguchi C, Katagi A, Kamitori K, Dong Y, Tsukamoto I, Tokuda M. 2015. Rare sugar D-psicose prevents progression and development of diabetes in T2DM model Otsuka Long-Evans Tokushima Fatty rats. Drug Des Devel Ther 9: 525-535.

14) Hossain A, Yamaguchi F, Matsuo T, Tsukamoto I, Toyoda Y, Ogawa M, Nagata Y, Tokuda M. 2015. Rare sugar D-allulose: Potential role and therapeutic monitoring in maintaining obesity and type 2 diabetes mellitus. Phar- 
macol Ther 155: 49-59.

15) Han Y, Han HJ, Kim AH, Choi JY, Cho SJ, Park YB, Jung UJ, Choi MS. 2016. D-Allulose supplementation normalized the body weight and fat-pad mass in diet-induced obese mice via the regulation of lipid metabolism under isocaloric fed condition. Mol Nutr Food Res 60: 1695-1706.

16) Yamada T, Iida T, Takamine S, Hayashi N, Okuma K. 2015. Safety evaluation of rare sugar syrup: single-dose oral toxicity in rats, reverse mutation assay, chromosome aberration assay, and acute non-effect level for diarrhea of a single dose in humans. Shokuhin Eiseigaku Zasshi (Food Hyg Saf Sci) 56: 211-216 (in Japanese).

17) Okuma K, Yamada K, Tsukuda K, Iida T, Oga H, Izumori K, Tsujisaka Y, Shimonishi T, Yamada T, Okamoto I, Inventor. 2008. Novel sweetener having sugar-like taste and production method and use of the same. Japan patent JP WO2008/142860 A1.

18) Iida T, Yamada T, Hayashi N, Okuma K, Izumori K, Ishii R, Matsuo T. 2013. Reduction of abdominal fat accumulation in rats by 8 -week ingestion of a newly developed sweetener made from high fructose corn syrup. Food Chem 138: 781-785.

19) Matsuo T, Baba Y, Hashiguchi M, Takeshita K, Izumori K, Suzuki H. 2001. Dietary D-psicose, a C-3 epimer of D-fructose, suppresses the activity of hepatic lipogenic enzymes in rats. Asia Pacific J Clin Nutr 10: 233-237.

20) Matsuo T, Baba Y, Hashiguchi M, Takeshita K, Izumori K, Suzuki H. 2001. Less body fat accumulation with D-psicose diet versus D-fructose diet. J Clin Biochem Nutr 30: $55-65$.

21) Folch J, Lees M, Sloane Stanley GH. 1957. A simple method for the isolation and purification of total lipides from animal tissues. J Biol Chem 226: 497-509.

22) Oku T, Murata-Takenoshita Y, Yamazaki Y, Shimura F,
Nakamura S. 2014. D-Sorbose inhibits disaccharidase activity and demonstrates suppressive action on postprandial blood levels of glucose and insulin in the rat. Nutr Res 34: 961-967.

23) Yamada T, Hayashi N, Iida T, Takamine S, Okuma K, Matsuo T. 2014. Dietary D-sorbose decreases serum insulin levels in growing Sprague-Dawley rats. J Nutr Sci Vitaminol 60: 297-299.

24) Lu Y, Levin GV, Donner TW. 2008. Tagatose, a new antidiabetic and obesity control drug. Diabetes Obes Metab 10: 109-134.

25) Bär A. 1999. Characteristics and significance of D-tagatose-induced liver enlargement in rats: An interpretative review. Regul Toxicol Pharmacol 29: S83-S93.

26) Murata A, Sekiya K, Watanabe Y, Yamaguchi F, Hatano N, Izumori K, Tokuda M. 2003. A novel inhibitory effect of D-allose on production of reactive oxygen species from neutrophil. J Biosci Bioeng 96: 89-91.

27) Nagata Y, Kanasaki A, Tamaru S, Tanaka K. 2015. D-Psicose, an epimer of D-fructose, favorably alters lipid metabolism in Sprague-Dawley rats. J Agric Food Chem 63: 3168-3176.

28) Ochiai M, Onishi K, Yamada T, Iida T, Matsuo T. 2014. D-Psicose increases energy expenditure and decreases body fat accumulation in rats fed a high-sucrose diet. Int J Food Sci Nutr 65: 245-250.

29) Yagi K, Matsuo T. 2009. The study on long-term toxicity of D-psicose in rats. J Clin Biochem Nutr 45: 271-277.

30) Tsukamoto I, Hossain A, Yamaguchi F, Hirata Y, Dong Y, Kamitori K, Sui L, Nonaka M, Ueno M, Nishimoto K, Suda H, Morimoto K, Shimonishi T, Saito M, Song T, Konishi R, Tokuda M. 2014. Intestinal absorption, organ distribution, and urinary excretion of the rare sugar D-psicose. Drug Des Devel Ther 8: 1955-1964. 